\title{
基于分段拟合的机床大尺寸工作台热误差补偿模型 ${ }^{*}$
}

\author{
张成新 ${ }^{1,2}$ 高 峰 ${ }^{1}$ 李 $^{\text {艳 }}{ }^{1}$ 赵柏涵 $^{1}$ 孟振华 $^{1}$ \\ (1. 西安理工大学机械及精密仪器工程学院 西安 710048;
}

2. 曲阜师范大学印刷学院 日照 276826)

\begin{abstract}
摘要: 大型机床工作台在往复运动过程中, 丝杜螺母会产生大量的热, 一部分热量从螺母传入工作台导致工作台两侧趐曲, 使工作台不同位置产生不同热误差。为提高大尺寸机床工作台的纵向热误差补偿精度, 提出分段拟合热误差建模预测方法。 该方法是沿工作台横向在多个位置建立对应点的纵向热误差模型, 然后由各点单模型预测值进行分段拟合建立工作台整体预 测模型, 利用分段拟合模型实现对工作台任意位置热误差预测。同时为了提高热误差模型预测精度和鲁棒性, 采用粒子群优 化算法根据实时反馈热误差数据对模型参数辨识, 使热误差模型能适应机床最新的工作状态。在一台三坐标铣床工作台上进 行试验, 建立 $X$ 轴快速运动时工作台纵向热误差模型, 试验结果表明: 该方法鲁棒性好预测精度高, 能够实现大尺寸工作台 任意位置的热误差补偿, 且具有一定的通用性。
\end{abstract}

关键词: 数控机床; 工作台; 热误差; 分段拟合; 实时辨识

中图分类号: TH161

\section{Model of Thermal Error Compensation of Large Size Worktable for Machine Tools Based on Piecewise Fitting}

\author{
ZHANG Chengxin ${ }^{1,2}$ GAO Feng ${ }^{1}$ LI Yan ${ }^{1}$ ZHAO Bohan ${ }^{1}$ MENG Zhenhua ${ }^{1}$ \\ (1. School of Mechanical and Precision Instrument Engineering, Xi'an University of Technology, Xi'an 710048;
}

2. School of Graphic Arts, Qufu Normal University, Rizhao 276826)

\begin{abstract}
When worktable is traveling along the $X, Y$, or $Z$ axis of machine tool back and forth, due to frictional interaction between moving parts, a great amount of heat is generated in ball-screw drive systems, and part of it is transferred to the worktable, by which two sides of the worktable parallel to the guide way will be warped. So the thermal deformations of various positions on the worktable are different. In order to improve the accuracy of thermal error compensation of large size worktable, a multi-location forecasting model with piecewise fitting compensation method is proposed. The corresponding thermal error model of the multi-point on the worktable is established, and piecewise fitting of multi-point prediction model is used to implement a thermal error compensation for whole worktable. Furthermore, to improve prediction accuracy and robustness of the thermal error model, particle swarm optimization algorithm is utilized to identify the model parameters based on the real-time feedback data, and thus the thermal error model could adapt to the latest working condition of the machine tools. The thermal error compensation experiment of the worktable in a three coordinate milling machine is carried out under the condition of $X$-axis's fast movement. The experimental results indicate that this method has a good robustness and a high precision, which can compensate thermal error of large size worktable at any position, and this method is of universal applicability.
\end{abstract}

Key words: $\mathrm{CNC}$ machine tools; worktable; thermal error; piecewise fitting; real-time identification

\section{0 前言}

在影响零件加工精度的因素中, 机床热误差是 影响加工精度的主要原因之一, 在精密机床加工中 由于热因素造成的加工误差可以占到 $60 \%$

\footnotetext{
* 国家自然科学基金(51375382)、国家科技重大专项(2012ZX04012032) 和陕西省科技厅(2013JM7014)资助项目。20140216 收到初稿, 20140827 收到修改稿
}

$70 \%{ }^{[1]}$ 。为降低或消除机床热误差对加工精度的影 响, 科研人员进行了大量广泛深入的研究 ${ }^{[2-4]}$, 目前 主要有两种减小热误差的途径: 硬件消除法和软件 补偿法 ${ }^{[2]}$ 。硬件消除法主要是通过热结构对称设计、 预拉伸和采用低热膨胀系数的材料来实现, 硬件消 除法缺点是成本较高。软件补偿法是通过建立能够 反映机床温度与热误差关系的数学模型, 在补偿时 用模型产生的预测值来抵消机床因温度变化产生的 误差, 以此达到消除热误差的目的。软件补偿法的 
优点是, 实现成本较低且无须对现有机床进行大的 改造, 应用简便易于推广。软件补偿法的关键是建 立能够准确反映机床温度与误差之间的数学模型, 这也是目前热误差补偿研究工作的热点和难点。补 偿模型分为离线静态模型和在线动态实时模型 ${ }^{[5]}$, 常见静态补偿模型如: 多元统计回归、最小二乘支 持向量机 ${ }^{[6]}$ 、灰色系统理论 ${ }^{[7]}$ 、神经网络 ${ }^{[8]}$ 等。如果 机床使用环境和建模环境相近, 静态热误差模型能 够取得较好的预测效果, 但是当机床使用环境和建 模环境相差比较大或环境温度和工况变化比较大 时, 模型预测精度会严重受到影响。为了提高热误 差补偿模型的精度和适应能力, 研究者提出了实时 在线动态补偿模型 ${ }^{[5,9-11]}$, 在线模型能够根据加工状 况、环境温度等的变化实时调整模型参数, 使模型 能跟踪机床热误差的变化, 所以在线动态模型具有 更高的预测精度和适应能力。

以上建模方法为热误差补偿提供了有力工具, 且在试验中取得了良好效果。机床部件受热不仅会 产生热膨胀伸长, 还会产生弯曲变形。如丝杜螺母 在传动过程中由于摩擦作用产生热, 文献[12]分析 了丝杜热弹性效应导致对工作台纵向定位精度的影 响。文献[13]提出一种几何误差和热误差建模方法, 对热误差分离后, 建立了不同位置不同温度下的热 误差补偿模型, 提高了工作台定位精度。实际工作 中丝杜螺母摩擦热一部分传入丝杜, 还有一部分传 入工作台，传入工作台的这部分热，分布不均匀将 会导致工作台热变形。现有文献在热误差建模时对 工作台热变形考虑较少, 本文在三坐标铣床上通过 试验发现丝杜螺母传入工作台的热导致工作台两侧 产生尧曲, 为了对工作台热变形造成的热误差进行 补偿, 先建立工作台不同位置热误差模型, 再对工 作台各点模型预测值进行分段拟合, 实现对工作台 任意位置的热误差预测。为了增加模型的预测精度 和适应能力, 通过聚类分析和逐步回归寻找最佳测 温点, 采用粒子群优化实时辨识动态模型参数, 提 高了模型鲁棒性和预测精度。

\section{1 温度测点的选取及建模方法}

在选取温度测点时认为不同位置温度点只要温 度变化规律相似, 对热误差模型的作用就相似, 在 建模时可以从相似的测点中选取一个作为代表变 量, 机床热误差模型可以用几个关键的温度点来表 征 $^{[14]}$ 。基于上述假设, 可以在机床上布置大量传感 器, 然后根据每点的温度变化规律进行分类, 再从 每个分类中选取一个温度变量进行热误差建模。这
样就减小了温度测点选取的盲目性, 且能利用较少 的温度变量建立热误差模型。本研究先用聚类分析 方法对温度变量进行分类, 再利用逐步回归选取与 热误差变化最相关的温度测点, 然后根据选取的测 点作为输入变量, 建立差分热误差模型。

\section{1 温度测点的选取方法}

(1) 聚类分析方法。聚类分析是一种分类方法, 它能将一批样品或变量, 按照它们在某种评定标准 上的相似程度或远近进行分类，目的是使同类内对 象的同质性最大和类与类之间的对象差异性最 $大^{[15]}$ 。为了对样品(变量)进行分类, 一般采用两种 方法: (1) 相似系数法, 性质越相似或越接近的它们 相似系数为 1 , 彼此无关的相似系数接近 0 , 相似的 样品(变量)归为一类; (2) 距离法, 把样品看做空间 中的一个点, 然后定义距离, 距离近的点归为一类。 常用的分类方法有 5 种: 即最短距离法、最长距离 法、类平均法、重心法、离差平方和法。

这 5 种聚类法的计算步骤一样, 不同的是距离 的定义方法, 当采用欧氏距离时 5 种方法有统一的 递推公式

$$
\begin{gathered}
D_{k r}^{2}=\alpha_{p} D_{k p}^{2}+\alpha_{q} D_{k q}^{2}+\beta D_{p q}^{2}+ \\
\gamma\left|D_{k p}^{2}-D_{k q}^{2}\right|
\end{gathered}
$$

式中, $D_{k r}$ 表示类 $G_{k}$ 与类 $G_{r}$ 之间的距离, $G_{r}$ 是 $G_{q}$ 和 $G_{p}$ 合并后形成的新类。 $\alpha_{p}, \alpha_{q}, \beta, \gamma$ 对不同方法有不 同的取值, 具体取值参见文献[15]。

采用系统聚类法进行分类的流程如图 1 所示。

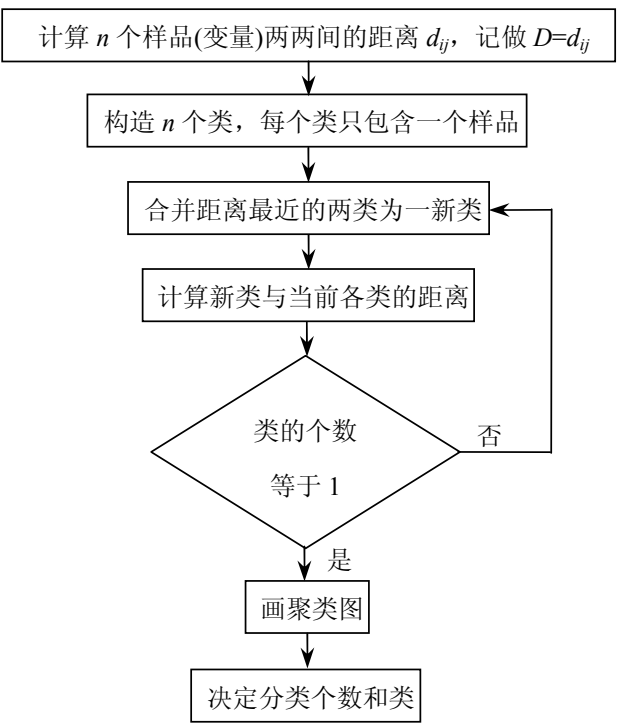

图 1 系统聚类流程图

（2）逐步回归原理。逐步回归原理是在建立因 变量和自变量回归模型时, 自变量逐次引入, 每引 入一个自变量, 对进入的变量进行逐个检验, 当原 
引入的变量由于后面变量的进入而变得不再显著 时, 就将其剔除。引入或剔除一个变量称为逐步回 归的一步, 每步都要进行 $F$ 检验, 以确保新引入变 量前, 回归方程中只包含显著地变量。这个过程反 复进行, 直到既无新的变量进入回归方程也无变量 可剔除, 计算停止得到逐步回归下的最优方程。

\section{2 建模原理}

(1) 差分方程模型。差分方程输出值不仅与当 前的输入值有关还和历史输入有关, 而热误差的变 化也与历史热输入大小有关, 因此采用差分结构描 述热膨胀更符合其物理意义。差分方程建模属于动 态建模, 差分方程形式如式(2)所示

$$
\begin{gathered}
E(k)+a_{1} E(k-1)+\cdots+a_{n} E(k-n)= \\
b_{0} T(k)+b_{1} T(k-1)+\cdots+b_{n} T(k-n)+w(k)
\end{gathered}
$$

式中, $k$ 表示采样时刻; $a, b$ 为方程系数; $n$ 为差分 方程的阶次; $E(k), T(K)$ 分别为系统的输出和输入 序列。

(2) 模型参数实时辨识方法。采用粒子群优化 算法, 根据实时反馈的温度和热误差数据对模型参 数进行辨识。

基于粒子群优化算法辨识原理：粒子群优化算 法(Particle swarm optimization, PSO $)^{[16-17]}$ 是一种群 体优化算法, 来源于人工生命和演化计算理论。基 本原理如下: 求解问题时, 每个粒子都随机设置初 始位置和初始速度, 初始位置表示在解空间中的一 组解, 速度表示搜索解空间的快慢。如在 $D$ 维空间 中搜索, 每个粒子就可以用 $D$ 维矢量表示, 第 $i$ 个 粒子的位置表示为 $x_{i}=\left(x_{i 1}, x_{i 2}, \cdots, x_{i D}\right)$, 速度表示为 $v_{i}=\left(v_{i 1}, v_{i 2}, \cdots, v_{i D}\right)$ 。每个粒子适应度的优劣由适应 度函数 $F$ 评价, 粒子经过一次迭代, 搜索到的个体 最优值: $p_{i}=\left(p_{i 1}, p_{i 2}, \cdots, p_{i D}\right)$, 群体最优值: $p_{g}=\left(p_{g 1}, p_{g 2}, \cdots, p_{g D}\right)$, 然后再跟据式(3)更新粒子位 置和速度

$$
\left\{\begin{array}{c}
v_{i d}(t+1)=v_{i d}(t)+c_{1} r_{1}\left(p_{i d}-x_{i d}(t)\right)+ \\
c_{2} r_{2}\left(p_{g d}-x_{i d}(t)\right) \\
x_{i d}(t+1)=x_{i d}(t)+v_{i d}(t+1)
\end{array}\right.
$$

式中, $i=1,2, \cdots, N ; d=1,2, \cdots, D ; c_{1}$ 和 $c_{2}$ 是非负常数; $r_{1}$ 和 $r_{2}$ 是介于 $[0,1]$ 的随机数; $v_{i d} \in\left[-v_{\text {max }}, v_{\text {max }}\right], v_{\text {max }}$ 为粒子最大速度; $t$ 为当前迭代次数。

PSO 算法流程图如图 2 所示。PSO 算法进行系 统辨识, 可以看成是一优化过程, 适应度函数取 如下

$$
\min (F)=\sum_{t=1}^{n}\left(y(t)-y_{0}(t)\right)^{2}
$$

式中, $y_{0}(t)$ 为实际系统输出; $y(t)$ 为模型输出, $\min$ 为取适应度函数的最小值。

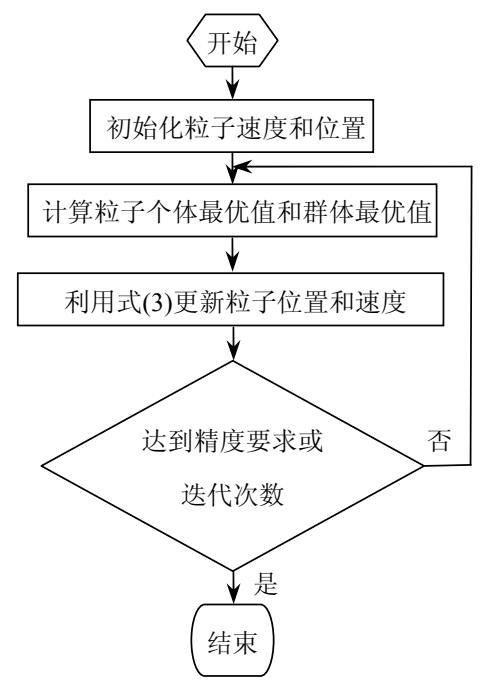

图 2 PSO 算法流程图

PSO 算法进行系统辨识是使一组粒子作为模型 参数, 使式(4)取得最小值, 对应的这组粒子就是待 求模型参数。

\section{2 热误差试验}

\section{1 试验安排}

本试验以三坐标铣床工作台为研究对象, $x$ 轴 以指令 $\mathrm{G} 00$ 快速移动时, 测量在工作台长度方向最 左、中、最右三个点纵向热变形(工作台尺寸：长 $900 \mathrm{~mm}$, 宽 $410 \mathrm{~mm}$ )。传感器安装如图 3 所示, $T_{1}$ 放置在丝杜螺母附近; $T_{2}, T_{3}$ 放置在伺服电机减速 器上; $T_{4}$ 放置在丝杜轴承座上; $T_{5}, T_{6}$ 放置在工作 台中间; $T_{7}$ 测量环境温度。电浴流位移传感器分别 安装在工作台最左、中、最右三个位置。工作台 $x$ 轴行程 $0 \sim 500 \mathrm{~mm}$; 往复移动速度(以指令 $\mathrm{G} 00$ 快 速运行); 第一个升温阶段快速运行 $2.5 \mathrm{~h}$, 停止 2.5 $\mathrm{h}$; 第二个升温阶段运行(第一阶段速度的 70\%)2.5 $\mathrm{h}$, 停止 $2.5 \mathrm{~h}$; 每隔 $5 \mathrm{~min}$ 对测点温度和热误差进行 一次采样。工作台运行 $1 \mathrm{~h}$ 后的丝杜螺母和工作台 的红外图像如图 4、5 所示; 温度和热误差测量数据 如图 6、7 所示。

图 4、5 表明, 工作台运动时丝杜螺母处温度比 较高, 局部可以达到 $40{ }^{\circ} \mathrm{C}$, 由此传入工作台使工 作台表面中间部位温度高, 两侧温度低, 工作台上 表面最高温度 $27{ }^{\circ} \mathrm{C}$, 说明从丝杜螺母处到工作台 上表面存在温度梯度。 


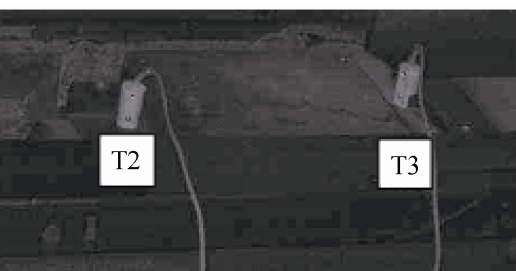

(a) 伺服电动机传感器布置

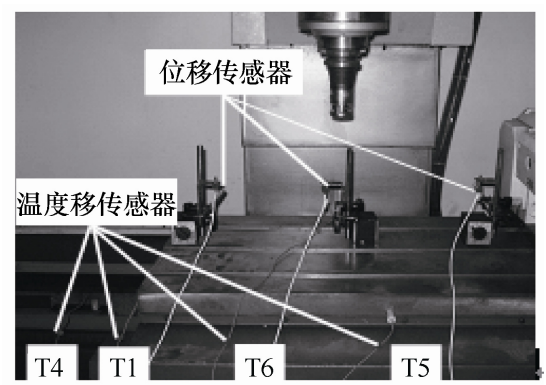

(b) 工作台位置传感器布置

图 3 传感器布置图

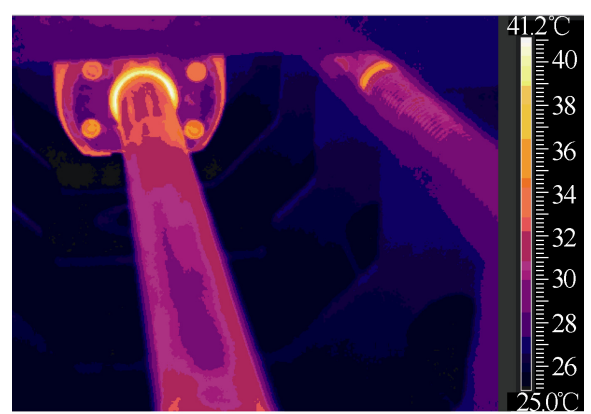

图 4 丝杜螺母红外图像

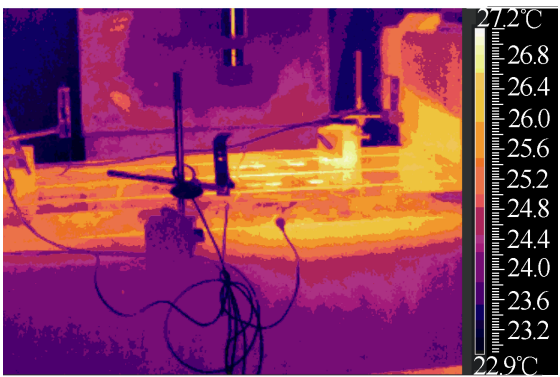

图 5 工作台红外像图

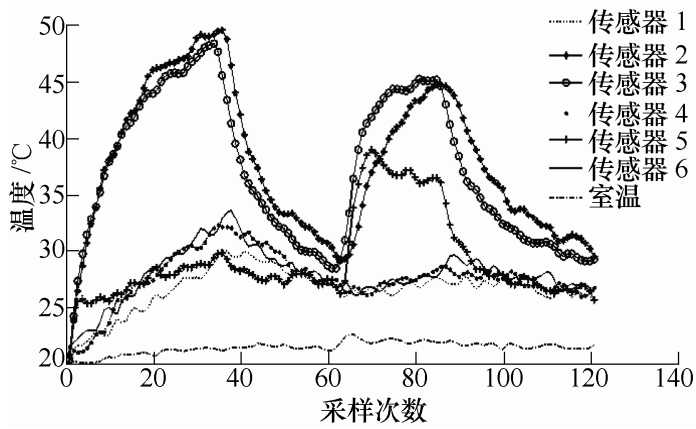

图 6 测量点温度变化曲线

2.2 试验结果定性分析

试验测量的温度和热误差曲线图 6、7 所示。从

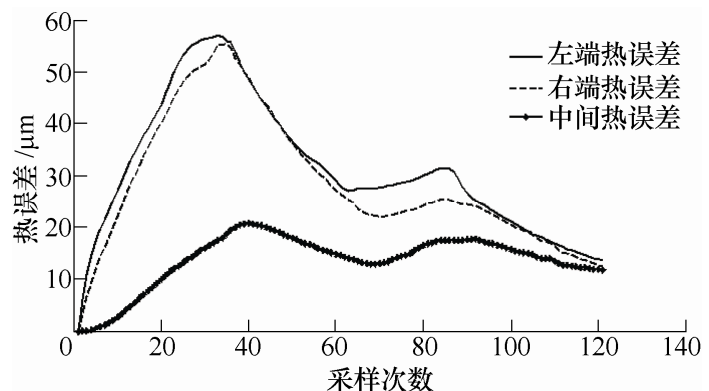

图 7 工作台热误差曲线

图 6 和图 7 看出, 当测点温度随机床工作状态变化 时, 工作台纵向误差也随之发生改变, 而且工作台 两侧和中间热误差变形量不同，也就是说工作台热 变形后呈 “凹状”。根据这一现象，对机床纵向加工 误差进行补偿时, 不能采取固定值, 而应该随着工 作台的位置不同而采取不同的补偿量使之适应工作 台面随温度和位置的变化。整个工作台随着受热变 形规律及变形量可由图 8 表示。从图 8 中看出工作 台在热变形时，两侧的热变形量要大于中间的热膨 胀量且两侧变形速度大于中间位置, 导致工作台两 侧翘曲，使台面呈凹状。

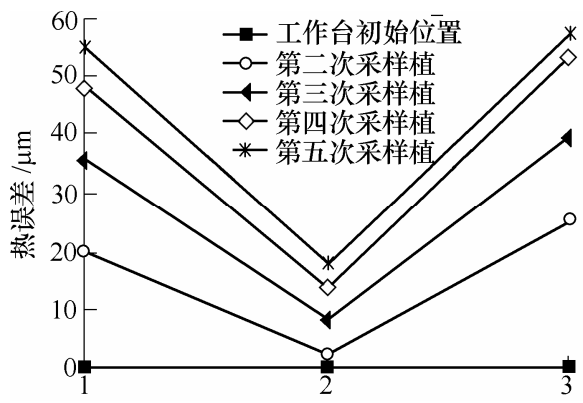

图 8 工作台变形示意图

1. 工作台最左侧 2. 工作台中间 3. 工作台最右侧

为了验证工作台在连续往复工作时由于热变形 呈 “凹状” 这一现象是否符合物理原理, 采用有限 元法进行验证(由于有限元仿真边界条件不可能完 全符合实际工作条件，仿真数据可能和实测数据有 差异，但是结果能够反映该变化的趋势，所以可以 利用有限元法进行验证这种现象), 如图 9 所示。有 限元热变形图是在丝杜螺母处给定摄氏温度 60 ${ }^{\circ} \mathrm{C}$, 仿真时间为 $20 \mathrm{~min}$ 的结果。从图 9 上能明显看 出工作台两侧发生翘曲, 与试验结果得到的现象相 吻合，说明试验观察到的现象是可能发生的。

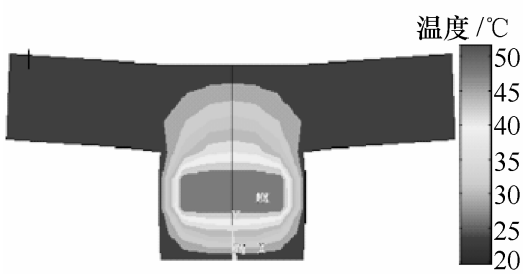

图 9 工作台热变形有限元分析示意图 


\section{3 温度测点选取及热误差建模}

\section{1 最佳温度测点的选取}

对试验测得 7 个温度变量, 先通过聚类分析对 温度变量的类型进行归类, 然后再利用逐步回归选 取和热误差有显著关系的温度变量。

对 7 个温度变量进行系统聚类分析, 采用最短
距离法, 平方 Euclidean 距离, 计算出两两变量的近 似矩阵, 如表 1 所示。

根据计算的近似系数，按图 1 流程得到图 10 所示聚类树, 纵坐标是变量个数, 横坐标聚合指数 是将实际的距离按照比例调整到 $0 \sim 25$ 的范围内。7 个温度变量按照四类进行选取，从每一类中选取 一个变量得到 $T_{1} 、 T_{5} 、 T_{7} 、 T_{2}$ 四个为基本待选 变量。

\section{表 1 近似矩阵}

\begin{tabular}{|c|c|c|c|c|c|c|c|}
\hline \multirow{2}{*}{ 变量 } & \multicolumn{7}{|c|}{ 量纲一平方 Euclidean 距离 } \\
\hline & 温度变量 1 & 温度变量 2 & 温度变量 3 & 温度变量 4 & 温度变量 5 & 温度变量 6 & 温度变量 7 \\
\hline 温度变量 1 & 0 & 19608.185 & 18042.277 & 233.383 & 2550.733 & 351.035 & 3574.987 \\
\hline 温度变量 2 & 19608.185 & 0 & 807.357 & 16121.491 & 13647.208 & 15406.042 & 37514.191 \\
\hline 温度变量 3 & 18042.277 & 807.357 & 0 & 14901.664 & 10823.384 & 14366.772 & 34438.570 \\
\hline 温度变量 4 & 233.383 & 16121.491 & 14901.664 & 0 & 2392.107 & 80.630 & 5206.652 \\
\hline 温度变量 5 & 2550.733 & 13647.208 & 10823.384 & 2392.107 & 0 & 2394.808 & 8809.072 \\
\hline 温度变量 6 & 351.035 & 15406.042 & 14366.772 & 80.630 & 2394.808 & 0 & 5577.717 \\
\hline 温度变量 7 & 3574.987 & 37514.191 & 34438.570 & 5206.652 & 8809.072 & 5577.717 & 0 \\
\hline
\end{tabular}

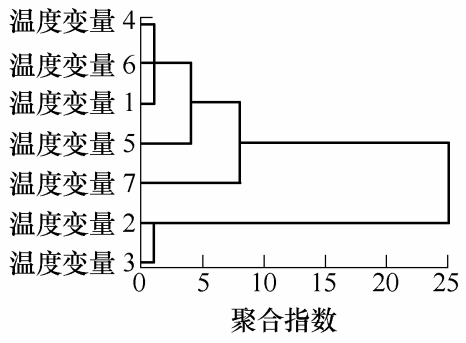

图 10 温度变量聚类树

对聚类分析得到的 $T_{1} 、 T_{2} 、 T_{5} 、 T_{7}$ 四个温度变 量和位移变量 $E$, 利用 SPSS 软件进行线性多元逐 步回归进行分析，得到回归方程

$$
E=-82.195+1.089 T_{1}+2.595 T_{2}
$$

方程复相关系数 $R=87 \%$, 各变量因子显著水平 远小于 0.05 , 说明各自变量与因变量线性相关程度 显著, 由此选取和热误差有显著关系的温度变量为 $T_{1}$ 和 $T_{2}$ 。

\section{2 差分方程阶次的确定}

确定方程阶次是采用从 1 阶逐次递增, 同时考 察模型预测值与测量值残差平方和的大小来确定, 当增加阶次残差平方和变化不明显时, 采用此时阶 次值。根据上面方法并考虑运算简单性, 确定差分 方程为二阶

$$
\begin{aligned}
& E(k)+a_{1} E(k-1)+a_{2} E(k-2)= \\
& b_{0} T_{1}(k)+b_{1} T_{1}(k-1)+b_{2} T_{1}(k-2)+ \\
& c_{0} T_{2}(k)+c_{1} T_{2}(k-1)+c_{2} T_{2}(k-2)
\end{aligned}
$$

由于工作台两侧和中间的变形量不一样, 因此 需要 3 个模型分别对工作台左侧、中间和右侧热误
差进行预测。如果工作台左右两侧热误差相差很小, 可以共用 1 个模型进行预测。

\section{3 参数模型实时辨识}

确定热误差模型结构后，采用粒子群优化算法 对模型参数进行辨识。对热误差模型参数辨识并不 是每个反馈周期都进行, 而是当模型输出值和热误 差反馈值残差超过一定范围时，再启动辨识算法对 模型参数进行辨识更新。辨识的输入数据为最近的 10 次采样数据, 使热误差模型始终反映机床最新的 工作状态。

\section{4 拟合模型的建立}

设 $E_{L}(t), E_{M}(t), E_{R}(t)$ 分别为工作台左侧、中间 和右侧在 $t$ 时刻的热误差模型预测值，为对工作台 左半部分行热误差预测, 利用 $E_{L}(t)$ 和 $E_{M}(t)$ 进行直 线拟合得到拟合公式(6), 由于不同时刻 $E_{L}(t)$ 和 $E_{M}(t)$ 取值不同，所以 $a$ 和 $b$ 是时变系数

$$
E(x)=a x+b
$$

式中, $x$ 为工作台位置, $a 、 b$ 为线性方程系数。

利用式(6)可以对工作台左侧部分, 进行任意位 置的纵向热误差预测。同理可以建立工作台右侧部 分的线性预测模型。

\section{4 热误差建模结果及分析}

\section{1 单点模型建模结果及分析}

由于工作台中间热误差模型和两侧热误差模 型结构形式相同, 辨识算法也一样, 在此只给出工 作台左侧热误差模型验证结果。采用粒子群优化参 
数辨识工作台左侧模型参数, 辨识结果如图 11 所示。

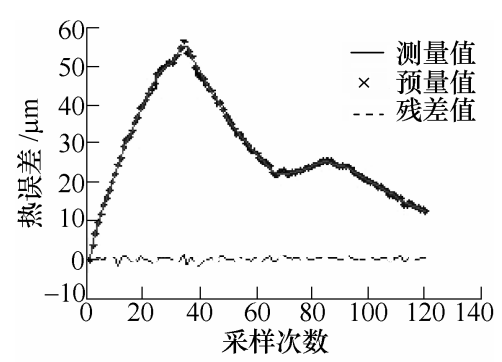

图 11 工作台左侧热误差建模结果

通过图 11 看出, 在第一个升温阶段模型预测值 与测量值符合较好残差很小, 因为此阶段工况单一, 热误差模型能够进行较好的预测; 当温度开始下降 时, 在第 38 个采样时刻出现一次波动, 说明热误差 模型参数和机床状态不匹配, 残差超出限定值需要 更新模型参数, 更新模型参数后直到下一个升温过 程, 预测值效果良好, 残差值较小。在第 68 个采样 时刻残差又出现一次波动, 模型进行参数更新, 使 热误差模型随着机床热状态的变化而实时调整。说 明该算法有一定的自适应能力, 能够根据残差的变 化实时更新模型参数, 模型预测结果如表 2 所示。

表 2 粒子群优化辨识模型预测结果 $\quad \mu \mathrm{m}$

\begin{tabular}{lllll}
\hline 残差指标 & 标准差 & 最大值 & 最小值 & 平方和 \\
\hline 水平 & 0.5013 & 1.5368 & 1.8213 & 40.4229 \\
\hline
\end{tabular}

\section{2 分段拟合模型验证及分析}

通过上述辨识过程得到工作台中间和两侧的 单点热误差模型, 要对工作台整体范围进行热误差 补偿, 还需要进一步对热误差模型进行扩展, 因为 上述热误差模型只是描述工作台三个位置的热误差 状态。为了对整个台面的热误差进行补偿, 对每一 时刻预测值得到的左、中和右三个数值进行分段拟 合得到拟合公式, 在补偿时由分段拟合公式得到工 作台任意位置的热误差量, 这样就可以做到对整个 工作台的任意位置进行补偿。为验证这一过程, 在 工作台中间传感器和最左端传感器中间处再放置一 个位移传感器, 用来测量该位置的热误差值作为验 证值。预测模型采用最左端和中间热误差模型的预 测值做直线拟合，然后根据得到的直线模型对中间 点进行预测。直线分段拟合模型预测值与测量值进 行比较, 结果如图 12 所示, 残差指标如表 3 所示。

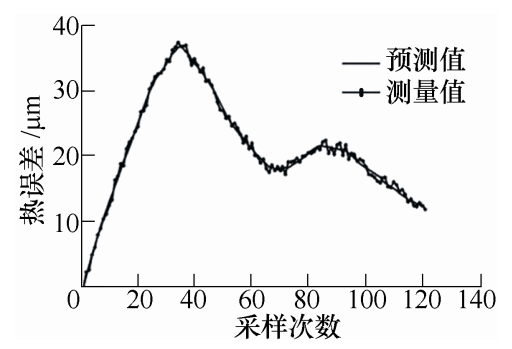

图 12 分段直线拟合模型预测结果结果图
表 3 分段直线拟合模型预测结果

\begin{tabular}{ccccc}
\hline 残差指标 & 标准差 & 最大值 & 最小值 & 平方和 \\
\hline 水平 & 0.5738 & 0.9910 & -0.9667 & 39.7917 \\
\hline
\end{tabular}

从图 12 和表 3 看出, 采用两点直线拟合公式 对中间热进行预测，效果良好，采用直线分段拟合 方法能够实现对工作台任意位置的纵向热误差精确 预测。根据此原理很容易建立工作台右侧部分的直 线分段拟合模型。

\section{5 结论}

(1) 通过系统聚类分析和逐步回归分析能够得 到热误差建模最佳测温点, 该方法简便易用。

(2) 通过粒子群优化算法, 能够根据反馈数据 对二阶差分模型参数进行实时辨识, 使模型能够跟 踪机床热误差的变化, 从而使模型保持较高的预测 精度。

(3) 试验中发现机床工作台两侧尧曲现象, 通 过对铣床工作台 $x$ 方向左、中、右三点分别建立热 误差模型, 再通过对三点热误差模型各时刻预测值 进行分段拟合, 实现对工作台任意位置热误差补偿。 为了对更大尺寸工作台进行热误差补偿, 只需要按 照此原理在工作台上布置更多的热误差传感器, 建 立对应点的热误差模型, 然后对各模型预测值进行 分段拟合得到整个工作台的纵向热误差补偿模型。 为了进一步提高多点模型拟合的精度, 可以采用高 次多项进行多点拟合。该方法为大尺寸工作台纵向 热误差补偿提供了一种新思路。

\section{参 考 文 献}

[1] RAMESH R, MANNAN M A, POO A N. Error compensation in machine tools - a review: Part II:

Thermal errors[J]. International Journal of Machine Tools and Manufacture, 2000, 40(9): 1257-1284.

[2] JOSEF M, JERZY J, ECKART U, et al. Thermal issues in machine tools[J]. CIRP Annals Manufacturing Technology, 2012, 61(2): 771-791.

[3] WECK M, MCKEOWN P, BONSE R, et al. Reduction and compensation of thermal errors in machine tools $[\mathrm{J}]$. CIRP Annals Manufacturing Technology, 1995, 44(2): 589-598.

[4] LI J W, ZHANG W J, YANG G S, et al. Thermal error modeling for complex physical systems: The-state-of-arts review $[\mathrm{J}]$. The International Journal of Advanced Manufacturing Technology, 2009, 42(1): 168-179.

[5] 杨建国, 任永强. 数控机床热误差补偿模型在线修正方 
法研究[J]. 机械工程学报, 2003，39(3): 81-84.

YANG Jianguo, REN Yongqiang. Research on-line modeling method of thermal eror compensation model for $\mathrm{CNC}$ machines[J]. Chinese Journal of Mechanical Engineering, 2003, 39(3): 81-84.

[6] 林伟青, 傅建中, 许亚洲, 等. 基于在线最小二乘支持 向量机的数控机床热误差建模与补偿 [J]. 计算机集成 制造系统，2008，14(2): 295-299.

LIN Weiqing, FU Jianzhong, XU Yazhou, et al. Thermal error modeling \& compensation of numerical control machine tools based on on-line least squares support vector machine[J]. Computer Integrated Manufacturing Systems, 2008, 14(2): 295-299.

[7] 问嘉钜, 杨建国. 灰色 $\mathrm{GM}(\mathrm{X}, \mathrm{N})$ 模型在数控机床热 误差建模中的应用[J]. 中国机械工程, 2009, 20(11): 1297-1300.

YAN Jiayu, YANG Jianguo. Application of grey GM(X, $\mathrm{N})$ model on $\mathrm{CNC}$ machine thermal error modeling $[\mathrm{J}]$. China Mechanical Engineering, 2009, 20(11): 1297-1300.

[8] 杨庆东. 神经网络补偿机床热变形误差的机器学习技 术 $[\mathrm{J}]$. 机械工程学报, 2000, 36(1): 92-95.

YANG Qingdong. Learning approach to compensation machine tools thermal defomation based on neural network[J]. Chinese Journal of Mechanical Engineering, 2000, 36(1): 92-95.

[9] 阳红, 向胜华, 刘立新, 等. 基于最优权系数组合建模 的数控机床热误差在线补偿 $[\mathrm{J}]$. 农业机械学报, 2012(5): 216-221.

YANG Hong, XIANG Shenghua, LIU Lixin, et al. Online compensation for $\mathrm{CNC}$ machine thermal error based on optimal weights based combined modeling[J]. Transactions of the Chinese Society for Agricaltural Machinery, 2012(5): 216-221.

[10] YANG H, NI J. Adaptive model estimation of machine-tool thermal errors based on recursive dynamic modeling strategy[J]. International Journal of Machine Tools and Manufacture, 2005, 45(1): 1-11.

[11] SHEN H, FU J, HE Y, et al. On-line asynchronous compensation methods for static/quasi-static error implemented on $\mathrm{CNC}$ machine tools[J]. International Journal of Machine Tools and Manufacture, 2012, 60: 14-26.

[12] 夏军勇, 胡友民, 吴波, 等. 热弹性效应分析与机床进
给系统热动态特性建模 [J]. 机械工程学报, 2010, 46(15): 191-198.

XIA Junyong, HU Youmin, WU Bo, et al. Analysis on thermoelastic domino effect and modeling on thermal dynamic characteristic of machine tools feed system[J]. Journal of Mechanical Engineering, 2010, 46(15): 191-198.

[13] 王维, 杨建国, 姚晓栋, 等. 数控机床几何误差与热误 差综合建模及其实时补偿[J]. 机械工程学报, 2012, 48(7): 165-170.

WANG Wei, YANG Jianguo, YAO Xiaodong, et al. Synthesis modeling and real-time compensation of geometric error and thermal error for $\mathrm{CNC}$ machine tools[J]. Journal of Mechanical Engineering, 2012 , 48(7): 165-170.

[14] RAGHUNATH V, MOSHE B, SHAW M C. Thermal effects on the accuracy of numerically controlled machine tools[J]. CIRP Annals-Manufacturing Technology, 1986, $35(1): \quad 255-258$.

[15] 于秀林, 任雪松. 多元统计分析[M]. 北京：中国统计 出版社, 1999.

YU Xiulin, REN Xuesong. Multivariate statistical analysis[M]. Beijing: China Statistics Press, 1999.

[16] 王峰, 邢科义, 徐小平. 系统辨识的粒子群优化方法 [J]. 西安交通大学学报，2009，43(2)：116-120.

WANG Feng, XING Keyi, XU Xiaoping. A system identification method using particle swarm optimization[J]. Journal of Xi'an Jiaotong University, 2009, 43(2): 116-120.

[17] 赵洋, 韦莉, 张逸成, 等. 基于粒子群优化的超级电容 器模型结构与参数辨识 $[\mathrm{J}]$. 中国电机工程学报, 2012, 32(15): 179-185.

ZHAO Yang, WEI Li, ZHANG Yicheng, et al. Structure and parameter identification of supercapacitors based on particle swarm optimization[J]. Proceeding of the CSEE, 2012, 32(15): 179-185.

作者简介: 张成新, 男, 1975 年出生, 博士研究生。主要研究方向为机 床热误差建模补偿。

E-mail: qfzcx_sd@163.com

高峰, 男, 1969 年出生, 教授, 博士研究生导师。主要研究方向为精密 制造, 大齿轮在机测量等。发表论文十余篇, 出版专著一部。

E-mail: gf2713@126.com 